\title{
Simple gradient flow equation for the bounce solution
}

\author{
Ryosuke Sato \\ Deutsches Elektronen-Synchrotron (DESY), Notkestraße 85, D-22607 Hamburg, Germany
}

(Received 8 July 2019; published 16 January 2020)

\begin{abstract}
Motivated by the recent work of Chigusa, Moroi, and Shoji, we propose a new simple gradient flow equation to derive the bounce solution which contributes to the decay of the false vacuum. Our discussion utilizes the discussion of Coleman, Glaser, and Martin, and we solve a minimization problem of the kinetic energy while fixing the potential energy. The bounce solution is derived as a scale transformation of the solution of this problem. We also show that the convergence of our method is robust against a choice of the initial configuration.
\end{abstract}

DOI: 10.1103/PhysRevD.101.016012

\section{INTRODUCTION}

The decay of the false vacua is an important topic in particle physics and cosmology. The decay rate of the false vacua can be calculated from "the imaginary part" of the Euclidean path integral [1]. ${ }^{1}$ In the path integral formalism, we can see that the main contribution comes from the bounce solution $\phi_{B}$, which is a nontrivial solution of the equation of motion with the least action. Thus, the bounce solution plays a crucial role in the decay of the false vacua. To calculate the bounce solution, we have to solve the equation of motion with the boundary condition at infinity. In general, it is not easy to calculate the bounce solution, and this is particularly the case for models with multiscalar fields.

Several algorithms to calculate the bounce action have been discussed so far-e.g., gradient flow with modifications [4-6], modified actions which have the bounce solution as a local minimum [7-10], gradually changing a coefficient of the friction term [the second term on the lhs of Eq. (7)] [11,12], machine learning $[13,14]$, and so on. Also, public codes to calculate the bounce solution are available, such as CosmoTransitions [15,16], AnyBubble [17], and BubbleProfiler [18,19]. Some works discuss the bounce solution or action avoiding the direct calculation-e.g., some approximations [20-22], upper bounds [23-25], lower bounds [25-27], and an alternative formulation [28-30].

One of the reasons for the technical difficulty is that the bounce solution is a saddle point of the action; i.e., the

${ }^{1}$ For earlier discussions, see, e.g., Refs. [2,3].

Published by the American Physical Society under the terms of the Creative Commons Attribution 4.0 International license. Further distribution of this work must maintain attribution to the author(s) and the published article's title, journal citation, and DOI. Funded by SCOAP ${ }^{3}$. bounce is not a stable solution of a simple minimization problem. Recently, Chigusa, Shoji, and Moroi [31] proposed a new method to obtain the bounce solution. They proposed a gradient flow equation whose fixed point is the bounce solution. Their flow equation has the gradient of the action and an additional term to lift up unstable direction around the bounce solution. Motivated by Ref. [31], in this paper, we propose a new simple flow equation. Coleman, Glaser, and Martin (CGM) [32] showed that the calculation of the bounce solution is equivalent to the minimization of the kinetic energy $\mathcal{T}$ while fixing the potential energy $\mathcal{V}<0$. This minimization problem can be naturally formulated in a flow equation. In the end, the bounce solution is obtained as a scale transformation of the solution of this problem. In Sec. II, we describe our formulation to calculate the bounce solution. In Sec. III, we discuss numerical analysis on several examples by using our flow equation, and show that our flow equation works well.

\section{FORMULATION}

In this paper, we focus on the Euclidean action with $n$ scalar fields with the canonical kinetic term.

$$
\begin{aligned}
& \mathcal{S}[\phi]=\mathcal{T}[\phi]+\mathcal{V}[\phi], \\
& \mathcal{T}[\phi]=\sum_{i=1}^{n} \int d^{d} x \frac{1}{2}\left(\nabla \phi_{i}\right)^{2}, \\
& \mathcal{V}[\phi]=\int d^{d} x V(\phi) .
\end{aligned}
$$

Here $d$ is the dimension of the space, and we assume $d$ is larger than 2. The scalar potential $V$ satisfies $V(0)=0$, $\partial V / \partial \phi_{i}=0$, all of the eigenvalues of the Hessian of $V$ at $\phi_{i}=0$ are non-negative, and $V$ is somewhere negative. 
The bounce solution which contributes to the decay of the false vacuum satisfies the equation of motion and the boundary condition at infinity:

$$
\begin{aligned}
-\nabla^{2} \phi_{i}+\frac{\partial V}{\partial \phi_{i}} & =0, \\
\lim _{|x| \rightarrow \infty} \phi_{i}(x) & =0 .
\end{aligned}
$$

Also, the bounce solution should be a nontrivial solution, i.e., $\exists i, x, \phi_{i}(x) \neq 0$. Thus,

$$
\mathcal{T}[\phi]>0, \quad \mathcal{V}[\phi]<0 .
$$

Note that $\mathcal{V}[\phi]<0$ is required in order for the bounce solution to be an extremum under the scale transformation: $\phi_{i}(x) \rightarrow \phi_{i}(\lambda x)$. See, e.g., Ref. [32]. The bounce solution has the least action among configurations which satisfy the above conditions [Eqs. (4), (5), and (6)]. It is known that the bounce solution has spherical symmetry [32-35]. Therefore, Eq. (4) can be simplified as

$$
-\frac{d^{2} \phi_{i}}{d r^{2}}-\frac{d-1}{r} \frac{d \phi_{i}}{d r}+\frac{\partial V}{\partial \phi_{i}}=0 .
$$

In order to discuss the bounce solution, CGM [32] introduced the reduced problem, which is defined as the problem of finding a configuration vanishing at infinity which minimizes $\mathcal{T}$ for some fixed negative $\mathcal{V}$. The existence of the solution of this problem is ensured by CGM's theorem B in Refs. [32,36]. Also, CGM's theorem A ensures that the bounce solution can be obtained as a scale transformation of a solution of the reduced problem. (See the Appendix.) Here we solve CGM's reduced problem by using a gradient flow equation. We introduce functions $\varphi_{i}(r, \tau)$ and propose the following gradient flow equations:

$$
\begin{aligned}
\frac{\partial}{\partial \tau} \varphi_{i}(r, \tau) & =\nabla^{2} \varphi_{i}-\lambda[\phi] \frac{\partial V(\varphi)}{\partial \varphi_{i}}, \\
\lambda[\varphi] & =\frac{\sum_{i} \int_{0}^{\infty} d r r^{d-1} \frac{\partial V(\varphi)}{\partial \varphi_{i}} \nabla^{2} \varphi_{i}}{\sum_{i} \int_{0}^{\infty} d r r^{d-1}\left(\frac{\partial V(\varphi)}{\partial \varphi_{i}}\right)^{2}} .
\end{aligned}
$$

Here $\tau$ is "the time" for the flow of $\varphi$ and $\nabla^{2} \varphi_{i}=$ $\partial_{r}^{2} \varphi_{i}+(d-1)\left(\partial_{r} \varphi\right) / r$. We take the initial $\varphi(r, 0)$ such that

$$
\left.\mathcal{V}[\varphi]\right|_{\tau=0}<0 .
$$

Note that $\lim _{r \rightarrow \infty} \varphi_{i}(r, \tau)=0$ should hold in order for $\mathcal{V}[\phi]$ to be finite. By using Eqs. (8) and (9), we can show

$$
\begin{aligned}
& \frac{d}{d \tau} \mathcal{V}[\varphi]=0, \\
& \frac{d}{d \tau} \mathcal{T}[\varphi] \leq 0 .
\end{aligned}
$$

To show Eq. (12), we used the following Cauchy-Schwarz inequality:

$$
\begin{aligned}
& \left(\sum_{i} \int_{0}^{\infty} d r r^{d-1}\left(\nabla^{2} \varphi_{i}\right)^{2}\right)\left(\sum_{i} \int_{0}^{\infty} d r r^{d-1}\left(\frac{\partial V(\varphi)}{\partial \varphi_{i}}\right)^{2}\right) \\
& \geq\left(\sum_{i} \int_{0}^{\infty} d r r^{d-1} \frac{\partial V(\varphi)}{\partial \varphi_{i}} \nabla^{2} \varphi_{i}\right)^{2} .
\end{aligned}
$$

Also, we can see that the equalities of Eqs. (12) and (13) hold if and only if

$$
\nabla^{2} \varphi_{i}=\lambda \frac{\partial V(\varphi)}{\partial \varphi_{i}}
$$

is satisfied. Equations (11) and (12) tell us that $\mathcal{T}[\varphi]$ monotonously decreases while $\mathcal{V}[\varphi]$ is constant during the flow of $\varphi$. In the limit of $\tau \rightarrow \infty, \varphi$ converges to a configuration which satisfies $\nabla^{2} \varphi_{i}-\lambda\left(\partial V(\varphi) / \partial \varphi_{i}\right)=0$. The convergence of $\varphi$ is guaranteed by the existence of the minimizer $[32,36]$. Note that this fixed point cannot be the false vacuum $\varphi_{i}=0$, because $\mathcal{V}[\varphi]$ in the neighborhood of the false vacuum is positive, and $\mathcal{V}[\varphi]$ is always negative during the flow. As long as the initial condition is not finetuned, $\varphi$ at $\tau \rightarrow \infty$ should be a stable solution under the small perturbation; i.e., $\mathcal{T}[\varphi]$ should be a local minimum under the small perturbation such that $\mathcal{V}[\phi]$ is not changed. In principle, the reduced problem could have several local minima. Physically, this case happens if there exist several directions of tunneling. In this case, $\varphi$ at $\tau \rightarrow \infty$ depends on the initial condition, and we can find the global minimum among those local minima. The configuration which gives the smallest value of $\mathcal{T}$ is the solution of the CGM's reduced problem.

Let $\phi_{i}(r)\left(\equiv \lim _{\tau \rightarrow \infty} \varphi_{i}(r, \tau)\right)$ be the solution of the reduced problem, and derive the bounce solution. The bounce solution $\phi_{B}(r)$ can be obtained by a scale transformation of $\phi$ as

$$
\phi_{B}(r)=\phi\left(\lambda^{1 / 2} r\right) .
$$

The above $\lambda$ is calculated as $\lim _{\tau \rightarrow \infty} \lambda[\varphi]$. Although CGM's theorem $\mathrm{A}$ ensures that this $\phi_{B}$ is the bounce solution, let us see this more explicitly. We can immediately see that (i) $\phi_{B}$ satisfies the EOM [Eq. (4)], and (ii) $\lim _{r \rightarrow \infty} \phi_{B}(r)=0$, because $\mathcal{V}\left[\phi_{B}\right]$ is finite. Also, we can see that (iii) $\mathcal{S}$ has only one unstable direction around $\phi_{B}$. Since $\phi_{B}$ is a scale transformation of $\phi, \phi_{B}$ is the global minimum of the action $\mathcal{S}$ if the potential energy $\mathcal{V}$ is fixed. The direction in which $\mathcal{S}$ decreases is the direction which changes $\mathcal{V}[\phi]$-i.e., the 
scale transformation. Therefore, $\phi_{B}$, which is defined in Eq. (15), is the bounce solution.

An essential point of our method is that the negative eigenmode around the bounce solution can be related to the scale transformation. By fixing the potential energy $\mathcal{V}$, we freeze fluctuation in this direction. Note that a method which is proposed in Ref. [4] also utilizes this property.

\section{EXAMPLE}

In the previous section, we have seen that the CGM's reduced problem can be solved by the flow equation [Eqs. (8) and (9)], and the bounce solution can be obtained from Eq. (15). In this section, we discuss numerical results for several examples, and show that our method works well.

First, let us take the following single scalar potential in $d=4$ Euclidean space:

$$
V(\phi)=\frac{1}{2} \phi^{2}-\frac{1}{3} \phi^{3} .
$$

We take the initial configuration at $\tau=0$ as

$$
\varphi(r, 0)=\left\{\begin{array}{ll}
5(1-r) & (0 \leq r \leq 1) \\
0 & (r>1)
\end{array} .\right.
$$

The flow of this field configuration is shown in Fig. 1. We can see the convergence of the configuration. In Figs. 2 and 3, we show the flow of the configuration from different initial conditions. We can see that the convergence of the configuration is robust for different initial conditions. The final configuration depends only on the value of $\mathcal{V}[\varphi]$, and those different final results are connected with each other by an appropriate scale transformation. By using this result, we can obtain the bounce solution from Eq. (15). We compare our bounce solution with the result by CosmoTransitions [16] in Fig. 4. We can see that the two results agree well, and our method works.

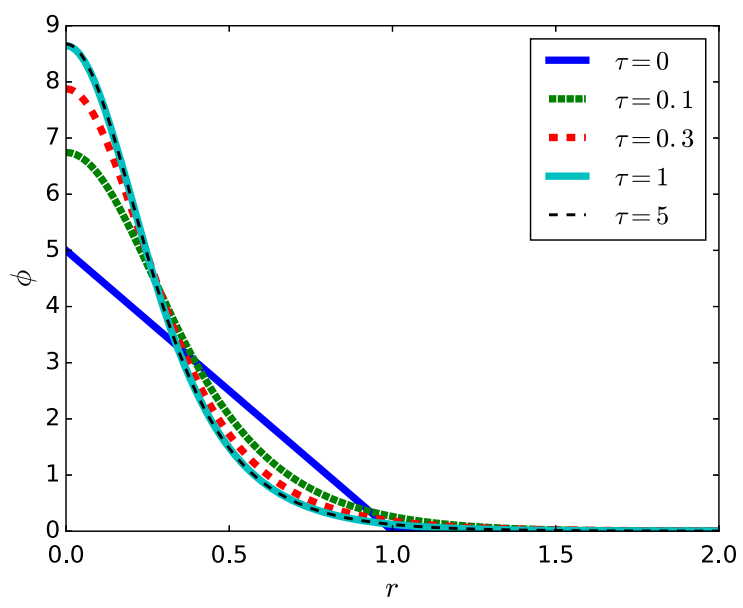

FIG. 1. A flow of the field configuration with the potential [Eq. (16)] with $d=4$ and the initial condition [Eq. (17)].

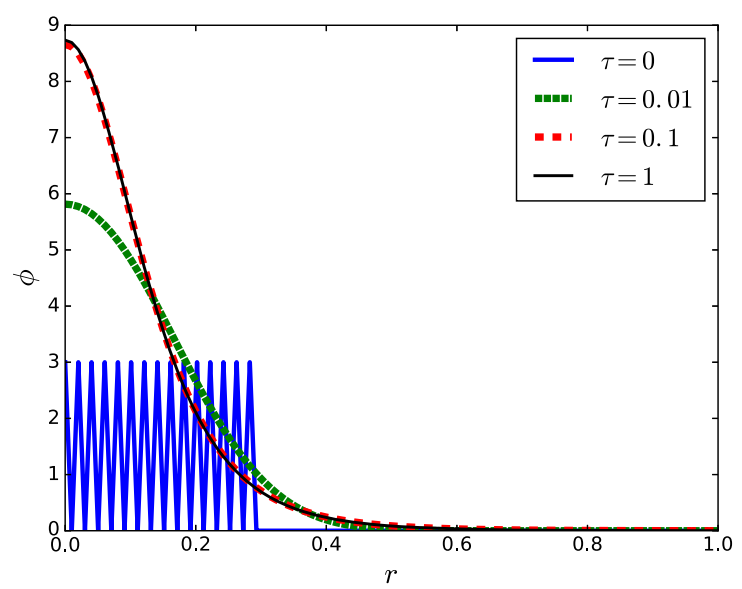

FIG. 2. Same as Fig. 1, except for the initial configuration.

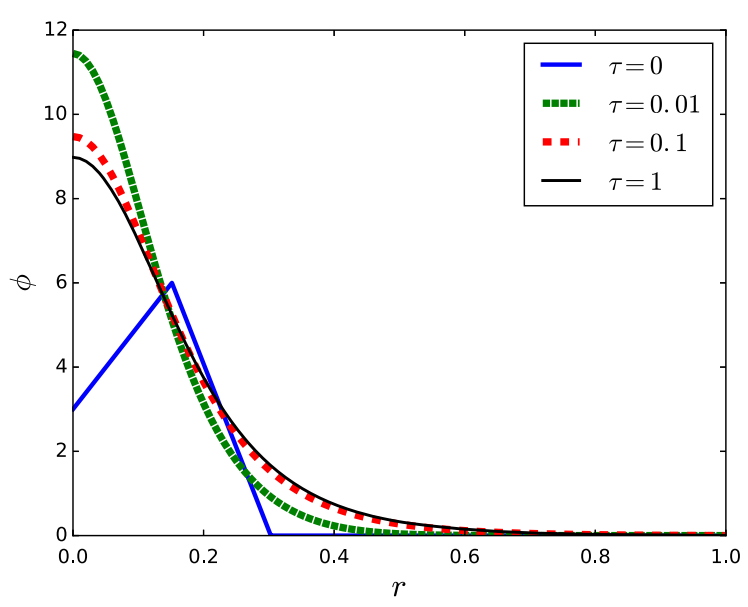

FIG. 3. Same as Fig. 1, except for the initial configuration.

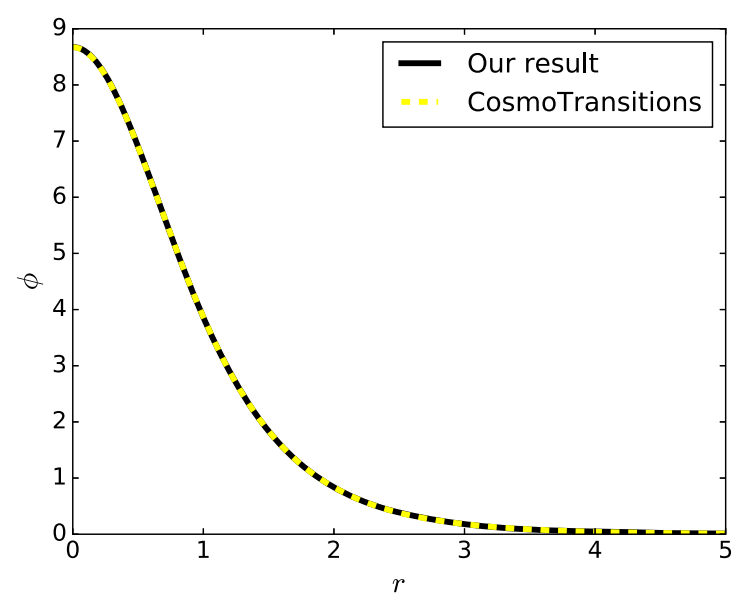

FIG. 4. The black line is obtained from Eq. (15) in the limit of large $\tau$. The yellow dotted line is calculated by CosmoTransitions. 


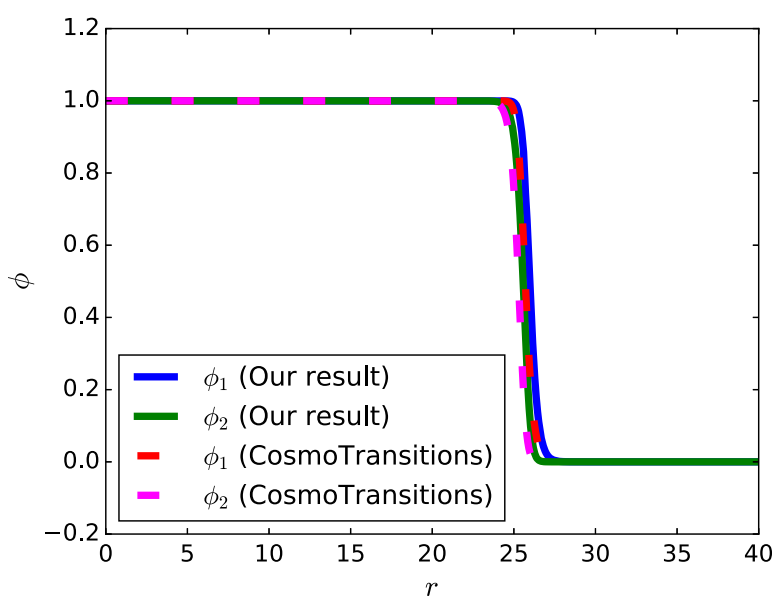

FIG. 5. The bounce solution in the $r-\phi$ plane is shown by solid lines. The dashed lines are results of cosmoTransitions. We take the potential [Eq. (18)] with $c=2$ in $d=4$ space.

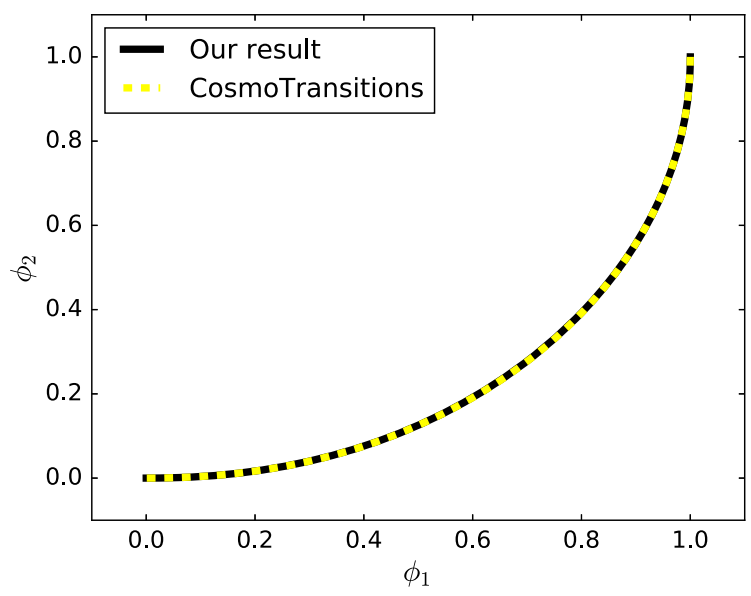

FIG. 6. The same bounce solution as Fig. 5 in the $\phi_{1}-\phi_{2}$ plane.

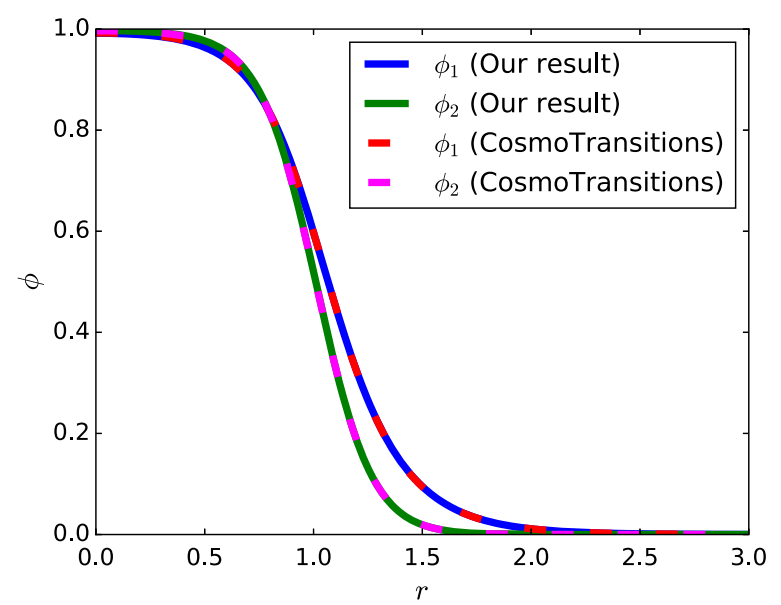

FIG. 7. The bounce solution in the $r-\phi$ plane is shown by solid lines. The dashed lines are results of cosmoTransitions. We take the potential Eq. (18) with $c=80$ in $d=4$ space.

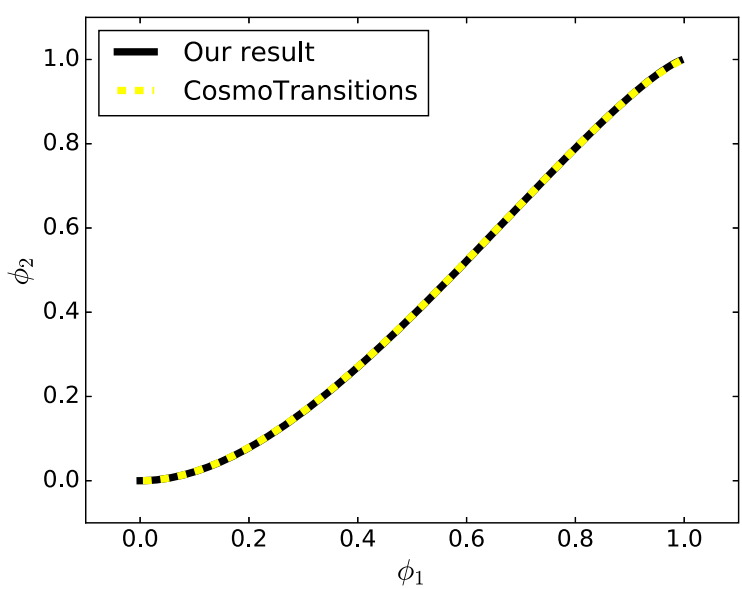

FIG. 8. The same bounce solution as Fig. 7 in the $\phi_{1}-\phi_{2}$ plane.

Next, let us discuss a case with two scalar fields. We take the following potential:

$V=\left(\phi_{1}^{2}+5 \phi_{2}^{2}\right)\left(5\left(\phi_{1}-1\right)^{2}+\left(\phi_{2}-1\right)^{2}\right)+c\left(\frac{1}{4} \phi_{2}^{4}-\frac{1}{3} \phi_{2}^{3}\right)$.

Again, we compare our bounce solutions with the results by CosmoTransitions. The case with $c=2$ is shown in Figs. 5 and 6 , and the case with $c=80$ in Figs. 7 and 8 . We can see that our result agrees with that of CosmoTransitions.

\section{CONCLUSION}

In this paper, motivated by a recent work of Chigusa, Shoji, and Moroi [31], we proposed a new simple gradient flow equation, which is defined in Eqs. (8) and (9). Our flow equation solves the CGM's reduced problem [32], i.e., the minimization problem of kinetic energy $\mathcal{T}$ while fixing potential energy $\mathcal{V}$. This minimization problem can be naturally formulated in a flow equation, and the bounce solution can be obtained as a scale transformation of this solution as Eq. (15). Since our flow equation solves the minimization problem and the existence of the minimizer is guaranteed by Refs. [32,36], the convergence of this method is robust against the choice of initial configuration as long as $\mathcal{V}[\varphi]<0$ is satisfied. A numerical package using this method is presented in Ref. [37]. ${ }^{2}$ This package calculates the Euclidean bounce action in $\mathcal{O}(0.1)$ s with $\mathcal{O}(0.1) \%$ accuracy for models with 1-8 scalar field(s), which is faster than CosmoTransitions [15,16], AnyBubble [17], and BubbleProfiler $[18,19]$.

\section{ACKNOWLEDGMENTS}

The author thanks Takeo Moroi for useful discussions.

\footnotetext{
${ }^{2}$ See also https://github.com/rsato64/SimpleBounce.
} 


\section{APPENDIX: CGM'S THEOREM A}

In this Appendix, we briefly summarize the theorem A in Ref. [32]. We denote the solution of the reduced problem for a given $\mathcal{V}$ as $\phi_{(\mathcal{V})}$. This theorem ensures that the bounce solution is given by a scale transformation of $\phi_{(\mathcal{V})}$.

$\phi_{\left(\mathcal{V}_{0}\right)}$ is a stationary point of $\mathcal{T}[\phi]+\lambda\left(\mathcal{V}[\phi]-\mathcal{V}_{0}\right)$, where $\lambda$ is the Lagrange multiplier. Thus, $\phi_{\left(\mathcal{V}_{0}\right)}$ satisfies

$$
-\nabla^{2} \phi_{\left(\mathcal{V}_{0}\right) i}+\lambda \frac{\partial V}{\partial \phi_{i}}=0
$$

Here, $\lambda$ should be appropriately chosen for the value of $\mathcal{V}_{0}$. We define the following configuration $\phi_{B}$ :

$$
\phi_{B}(x)=\phi_{\left(\mathcal{V}_{0}\right)}\left(\lambda^{1 / 2} x\right) .
$$

We can see that this is the bounce solution. First, by using Eqs. (A1) and (A2), we can check that $\phi_{B}$ satisfies the EOM [Eq. (4)]. Next, let us show that the action of any nontrivial solution of Eq. (4) is equal to or larger than $\mathcal{S}\left[\phi_{B}\right]$. Let $\tilde{\phi}$ be a nontrivial solution of Eq. (4). The action of $\tilde{\phi}$ is extremized under the scale transformation of $\tilde{\phi}$. Therefore,

$$
(d-2) \mathcal{T}[\tilde{\phi}]+d \mathcal{V}[\tilde{\phi}]=0 .
$$

There exists a solution of the reduced problem for $\mathcal{V}=\mathcal{V}[\tilde{\phi}]$, and the kinetic energy is not larger than $\mathcal{T}[\tilde{\phi}]$ :

$$
\mathcal{T}\left[\phi_{(\mathcal{V}[\tilde{\phi}])}\right] \leq \mathcal{T}[\tilde{\phi}]
$$

$\mathcal{T}\left[\phi_{B}\right]$ and $\mathcal{V}\left[\phi_{B}\right]$ are given as

$$
\begin{aligned}
& \mathcal{T}\left[\phi_{B}\right]=\lambda^{1-d / 2} \mathcal{T}\left[\phi_{(\mathcal{V}[\phi]}\right], \\
& \mathcal{V}\left[\phi_{B}\right]=\lambda^{-d / 2} \mathcal{V}[\phi] .
\end{aligned}
$$

Here, $\lambda \geq 1$ because of $(d-2) \mathcal{T}\left[\phi_{B}\right]+d \mathcal{V}\left[\phi_{B}\right]=0$ and Eqs. (A3) and (A4). Thus, by using Eqs. (A4) and (A5), we can show that

$$
\mathcal{T}\left[\phi_{B}\right] \leq \mathcal{T}[\tilde{\phi}] .
$$

$\mathcal{S}=(2 / d) \mathcal{T}$ is satisfied for solutions of Eq. (4). Then,

$$
\mathcal{S}\left[\phi_{B}\right] \leq \mathcal{S}[\tilde{\phi}]
$$

Thus, $\phi_{B}$ has the least action among the nontrivial solutions of the EOM.
[1] S. R. Coleman, The fate of the false vacuum: 1. Semiclassical theory, Phys. Rev. D 15, 2929 (1977); Erratum, Phys. Rev. D16, 1248(E) (1977).

[2] T. D. Lee and G. C. Wick, Vacuum stability and vacuum excitation in a spin 0 field theory, Phys. Rev. D 9, 2291 (1974).

[3] P. H. Frampton, Vacuum Instability and Higgs Scalar Mass, Phys. Rev. Lett. 37, 1378 (1976); Erratum, Phys. Rev. Lett.37, 1716(E) (1976).

[4] M. Claudson, L. J. Hall, and I. Hinchliffe, Low-energy supergravity: False vacua and vacuous predictions, Nucl. Phys. B228, 501 (1983).

[5] J. M. Cline, J. R. Espinosa, G. D. Moore, and A. Riotto, String mediated electroweak baryogenesis: A critical analysis, Phys. Rev. D 59, 065014 (1999).

[6] J. M. Cline, G. D. Moore, and G. Servant, Was the electroweak phase transition preceded by a color broken phase?, Phys. Rev. D 60, 105035 (1999).

[7] A. Kusenko, Improved action method for analyzing tunneling in quantum field theory, Phys. Lett. B 358, 51 (1995).

[8] A. Kusenko, P. Langacker, and G. Segre, Phase transitions and vacuum tunneling into charge and color breaking minima in the MSSM, Phys. Rev. D 54, 5824 (1996).
[9] J. M. Moreno, M. Quiros, and M. Seco, Bubbles in the supersymmetric standard model, Nucl. Phys. B526, 489 (1998).

[10] P. John, Bubble wall profiles with more than one scalar field: A numerical approach, Phys. Lett. B 452, 221 (1999).

[11] T. Konstandin and S. J. Huber, Numerical approach to multi dimensional phase transitions, J. Cosmol. Astropart. Phys. 06 (2006) 021.

[12] J.-H. Park, Constrained potential method for false vacuum decays, J. Cosmol. Astropart. Phys. 02 (2011) 023.

[13] R. Jinno, Machine learning for bounce calculation, arXiv: 1805.12153.

[14] M. L. Piscopo, M. Spannowsky, and P. Waite, Solving differential equations with neural networks: Applications to the calculation of cosmological phase transitions, Phys. Rev. D 100, 016002 (2019).

[15] S. Profumo, L. Ubaldi, and C. Wainwright, Singlet scalar dark matter: Monochromatic gamma rays and metastable vacua, Phys. Rev. D 82, 123514 (2010).

[16] C. L. Wainwright, CosmoTransitions: Computing cosmological phase transition temperatures and bubble profiles with multiple fields, Comput. Phys. Commun. 183, 2006 (2012). 
[17] A. Masoumi, K. D. Olum, and B. Shlaer, Efficient numerical solution to vacuum decay with many fields, J. Cosmol. Astropart. Phys. 01 (2017) 051.

[18] S. Akula, C. Balázs, and G. A. White, Semi-analytic techniques for calculating bubble wall profiles, Eur. Phys. J. C 76, 681 (2016).

[19] P. Athron, C. Balázs, M. Bardsley, A. Fowlie, D. Harries, and G. White, BubbleProfiler: Finding the field profile and action for cosmological phase transitions, Comput. Phys. Commun. 244, 448 (2019).

[20] M. C. Johnson and M. Larfors, Field dynamics and tunneling in a flux landscape, Phys. Rev. D 78, 083534 (2008).

[21] A. Masoumi, K. D. Olum, and J. M. Wachter, Approximating tunneling rates in multi-dimensional field spaces, J. Cosmol. Astropart. Phys. 10 (2017) 022.

[22] V. Guada, A. Maiezza, and M. Nemevšek, Multifield polygonal bounces, Phys. Rev. D 99, 056020 (2019).

[23] I. Dasgupta, Estimating vacuum tunneling rates, Phys. Lett. B 394, 116 (1997).

[24] U. Sarid, Tools for tunneling, Phys. Rev. D 58, 085017 (1998).

[25] A. R. Brown, Thin-wall approximation in vacuum decay: A lemma, Phys. Rev. D 97, 105002 (2018).

[26] A. Aravind, D. Lorshbough, and S. Paban, Lower bound for the multifield bounce action, Phys. Rev. D 89, 103535 (2014).

[27] R. Sato and M. Takimoto, Absolute Lower Bound on the Bounce Action, Phys. Rev. Lett. 120, 091802 (2018).
[28] J. R. Espinosa, A fresh look at the calculation of tunneling actions, J. Cosmol. Astropart. Phys. 07 (2018) 036.

[29] J. R. Espinosa, A fresh look at the calculation of tunneling actions including gravitational effects, Phys. Rev. D 100, 104007 (2019).

[30] J. R. Espinosa and T. Konstandin, A fresh look at the calculation of tunneling actions in multi-field potentials, J. Cosmol. Astropart. Phys. 01 (2019) 051.

[31] S. Chigusa, T. Moroi, and Y. Shoji, Bounce configuration from gradient flow, Phys. Lett. B 800, 135115 (2020).

[32] S. R. Coleman, V. Glaser, and A. Martin, Action minima among solutions to a class of Euclidean scalar field equations, Commun. Math. Phys. 58, 211 (1978).

[33] O. Lopes, Radial symmetry of minimizers for some translation and rotation invariant functionals, J. Differ. Equations 124, 378 (1996).

[34] J. Byeon, L. Jeanjean, and M. Mariş, Symmetry and monotonicity of least energy solutions, Calculus Var. Partial Differ. Equations 36, 481 (2009).

[35] K. Blum, M. Honda, R. Sato, M. Takimoto, and K. Tobioka, $\mathrm{O}(N)$ invariance of the multi-field bounce, J. High Energy Phys. 05 (2017) 109; Erratum, J. High Energy Phys.060(E) (2017) 60.

[36] H. Brezis and E. H. Lieb, Minimum action solutions of some vector field equations, Commun. Math. Phys. 96, 97 (1984).

[37] R. Sato, SimpleBounce: A simple package for the false vacuum decay, arXiv:1908.10868. 